\title{
Deleting multiple lytic genes enhances biomass yield and production of recombinant proteins by Bacillus subtilis
}

\author{
Yi Wang ${ }^{1}$, Zhenmin Chen ${ }^{2,3}$, Ruili Zhao ${ }^{1}$, Tingting Jin ${ }^{1}$, Xiaoming Zhang ${ }^{1}$ and Xiangdong Chen ${ }^{1,3^{*}}$
}

\begin{abstract}
Background: Bacillus subtilis is widely used in agriculture and industrial biotechnology; however, cell autolysis significantly decreases its yield in liquid cultures. Numerous factors mediate the lysis of $B$. subtilis, such as cannibalism factors, prophages, and peptidoglycan (PG) hydrolases. The aim of this work was to use molecular genetic techniques to develop a new strategy to prevent cell lysis and enhance biomass as well as the production of recombinant proteins.

Results: Five genes or genetic elements representing three different functional categories were studied as follows:

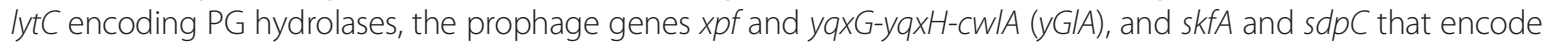
cannibalism factors. Cell lysis was reduced and biomass was enhanced by deleting individually $s k f A, s d p C$, xpf, and lytC. We constructed the multiple deletion mutant LM2531 ( $\mathrm{kfA}$ sdpC lytC xpf) and found that after $4 \mathrm{~h}$ of culture, its biomass yield was significantly increased compared with that of prototypical B. subtilis 168 (wild-type) strain and that $15 \%$ and $92 \%$ of the cells were lysed in cultures of LM2531 and wild-type, respectively. Moreover, two expression vectors were constructed for producing recombinant proteins ( $\beta$-galactosidase and nattokinase) under the control of the P43 promoter. Cultures of LM2531 and wild-type transformants produced $13741 \mathrm{U} / \mathrm{ml}$ and $7991 \mathrm{U} / \mathrm{ml}$ of intracellular $\beta$-galactosidase, respectively (1.72-fold increase). Further, the level of secreted nattokinase produced by strain LM2531 increased by 2.6-fold compared with wild-type (5226 IU/ml vs. $2028 \mathrm{IU} / \mathrm{ml}$, respectively).

Conclusions: Our novel, systematic multigene deletion approach designed to inhibit cell lysis significantly increased the biomass yield and the production of recombinant proteins by $B$. subtilis. These findings show promise for guiding efforts to manipulate the genomes of other $B$. subtilis strains that are used for industrial purposes.
\end{abstract}

Keywords: Bacillus subtilis, Cell lysis, Biomass, Recombinant protein, Multiple gene silencing, Nattokinase

\section{Background}

Bacillus subtilis and related species such as Bacillus licheniformis and Bacillus megaterium are designated generally recognized as safe (GRAS) microorganisms [1]. Probiotic preparations of B. subtilis are commercially available worldwide as nutritional supplements and are used for preventing and treating gastrointestinal disorders such as diarrhea $[2,3]$. Further, B. subtilis is used to produce agents for treating plant and animal diseases, which may replace chemical germicides that are toxic to humans and

\footnotetext{
* Correspondence: xdchen@whu.edu.cn

'State Key Laboratory of Virology, College of Life Sciences, Wuhan University, Wuhan 430072, China

${ }^{3}$ Hubei Provincial Cooperative Innovation Center of Industrial Fermentation, Wuhan 430072, China

Full list of author information is available at the end of the article
}

other species and harm the environment [4-6]. Moreover, B. subtilis strains are major workhorses of industrial biotechnology and are widely employed as cell factories to produce useful enzymes such as proteases, amylases, and hydrolases as well as biochemicals such as riboflavin, nucleotides, nonribosomal peptides, antibiotics, and insecticides [7-9]. In particular, the ability of B. subtilis to secrete proteinaceous products into the growth medium greatly facilitates downstream processing, which is highly advantageous for industrial-scale fermentation [1].

Cell lysis occurs in liquid cultures of B. subtilis, which may significantly decrease the total biomass and reduce the fermentation yields. Therefore, we investigated whether inhibiting cell lysis increases biomass. B. subtilis genes involved in cell lysis are roughly divided into three categories [10-12]. The first category includes genes encoding 
products that play roles in cannibalism during sporulation. Nutrient limitation triggers the activation of Spo0A, the main regulator of sporulation, in a portion of cells. Spo0A regulates the $s k f$ and $s d p$ operons. A small peptide encoded by $s k f A$, the first gene of the $s k f$ operon, acts as an antibiotic that kills siblings. The products of $s k f E$ and $s k f F$ export the peptide antibiotic and confer resistance to it. Cells that do not express spoOA are killed, and their contents provide nutrients that feed their siblings and delay sporulation of the entire bacterial population. In cells that express Spo0A, the $s d p$ operon produces the signaling protein $\mathrm{SdpC}$ that increases the killing effect [13-15].

Published studies mainly focus on the mechanism and biological significance of cannibalization; however, its effect on the biomass of liquid cultures of B. subtilis is unknown. Interestingly, the $s k f$ operon resides within prophage 1 [16], which may represent a defective viral genomic remnant. The second category of genes that influence the biomass of $B$. subtilis cultures includes prophages that can be activated and induce cell lysis. The B. subtilis genome harbors at least ten putative intact or partial prophages, and only PBSX and SP $\beta$ are lysogenic [10,17]. Heat shock treatment of the $\mathrm{SP} \beta c 2$ mutant of $B$. subtilis 168 (wild-type) induces the production of high titers of SP $\beta$ [18]. However, this prophage is difficult to induce in wild-type B. subtilis, including the prototype strain 168 , because the $c$ gene encodes a strong repressor of SP $\beta$ induction $[19,20]$. The prophage PBSX exists in most of B. subtilis strain and it is induced by treating cells with agents that cause Survival-Oriented-Behavior responses, such as mitomycin C, hydrogen peroxide, UV irradiation, and thymidine starvation. Upon induction, random DNA fragments of approximately $13 \mathrm{~kb}$ derived from various sites of the host chromosome are packaged by PBSX proteins, and these defective phage particles are released by lysis of the host [21].

Induction of PBSX in normal bacterial cultures is inconspicuous, because only defective phage particles are released. Moreover, PBSX particles and subvirion components are detected in normal cultures of B. subtilis [22], suggesting that PBSX may be constitutively activated in a small percentage of cells during bacterial growth and consequently reduce cell density. The other eight prophages, including prophage 1 , are considered chromosomal prophage remnants, because they lack the ability to replicate [17]. However, at least one PG hydrolase encoded by the lysis gene skin ( $c w 1 A)$, hydrolyzes the B. subtilis cell wall in vitro [23], indicating that these inactive prophages may influence the yield of bacterial biomass.

The third category of genes that mediate cell lysis encode PG hydrolases that are expressed during vegetative growth. PG (or murein) is a continuous covalent heteropolymer that extends to the outer side of the cytoplasmic membrane of most bacteria [11]. PG hydrolases mediate functions such as cell growth, cell separation, cell wall turnover, cell mobility, sporulation, and germination [10] and include the vegetative cell wall hydrolases LytC (CwlB), LytD (CwlG) [24], LytE (CwlF) [25], LytF (CwlE) [26], LytG (YubE) [27], CwlO (YvcE) [28], CwlS (YojL) [29] and CwlK (YcdD) [30]. Only LytC, LytD, LytG, and LytE have been proven to mediate cell lysis [10,27]. LytC and LytD possess amidase and $N$-acetylglucosaminidase activities, respectively, and transcription of the both genes is controlled by the sigma factor $\sigma^{\mathrm{D}}$. LytC is considered as the critical PG hydrolase that mediates autolysis of vegetative cells, because individual deletion of $l y t C$ can effectively inhibit cell lysis $[24,31]$. LytD seems to influence cell lysis when accompanying with LytC, since disruption of only $l y t D$ does not significantly affect sodium azideinduced cell lysis, but a lytC lytD double mutant exhibits less cell lysis than a lytC single mutant [24]. Similar to lytD, lyt $G$ encodes another $N$-acetylglucosaminidase, which is also a minor PG hydrolases [27]. Meanwhile, another lysis gene $l y t E$ encodes an endopeptidase. Single mutant of $l y t E$ displayed a growth defect and cell lysis reduction. However, LytE had no significant effect on the degradation of isolated cell wall preparations and was predicted to facilitate the action of LytC [25]. Further, a lytE cwlO double mutant undergoes synthetic lethality for the defect in lateral cell wall metabolism and cell elongation [32,33].

Here, we describe the development of a molecular genetics approach designed to delete multiple genes involved in cell lysis to improve biomass and the production of recombinant proteins. The results show that individually deleting $s k f A, s d p C$, xpf [34], or lytC decreased cell lysis and increased biomass compared with wild-type. Deleting different combinations of these genes further increased biomass, and a quadruple mutant produced a high level of biomass and least cell lysis. Moreover, higher levels of $\beta$-galactosidase and nattokinase were produced by the quadruple mutant. In contrast, there was no detectable effect on $\beta$-galactosidase or nattokinase production by a mutation of $y G l A$, the $y q x G-y q x H$-cwlA gene cluster related to the hydrolase of phage skin. These findings may be relevant for manipulating other industrial Bacillus strains that ubiquitously harbor homologous genes.

\section{Results}

Analysis of biomass of $B$. subtilis strains harboring mutations in individual genes encoding proteins involved in cell lysis

To test the influence of targeted mutation of genes involved in cell lysis on the biomass of batch cultures of B. subtilis, we generated the deletion mutants LM1 (xpf), LM2 (skfA), LM3 (lytC), LM4 ( $y G l A)$, and LM5 ( $s d p C)$, respectively (Additional file 1: Table S1, Appendix S1). The mutants and wild-type were inoculated into LB 
medium and cultured in $250-\mathrm{mL}$ flasks at $37^{\circ} \mathrm{C}$ with shaking for $12 \mathrm{~h}$. Consistent with published data [35], the transition of wild-type from exponential growth to stationary phase occurred between 6 to $7 \mathrm{~h}$, after which the optical density measured at $600 \mathrm{~nm}\left(\mathrm{OD}_{600}\right)$ decreased, indicating autolysis (Figure 1a). The growth rates of the wild-type and five mutants were not significantly different before $7 \mathrm{~h}$. However, there was a noticeable difference among these strains immediately after they entered stationary phase $(7-12 \mathrm{~h})$. For example, the $\mathrm{OD}_{600}$ values of cultures of strains LM2 (skfA), LM3 (lytC), and LM5 $(s d p C)$ continued to increase after $7 \mathrm{~h}$ and were approximately twice that of wild-type at $12 \mathrm{~h}$. The $\mathrm{OD}_{600}$ of the $x p f$ mutant culture was higher than that of wild-type after $12 \mathrm{~h}$. Only the growth of LM4 $(y G l A)\left(\mathrm{OD}_{600}=4.2\right)$ was similar to that of the wild-type $\left(\mathrm{OD}_{600}=3.7\right)$ at $12 \mathrm{~h}$, indicating that the PG hydrolase encoded by skin may not contribute to cell lysis.

Consistent with the $\mathrm{OD}_{600}$ data, the number of viable cells (colony forming units) in cultures of LM2 (skfA) and LM5 $(s d p C)$ were twice that of wild-type (Figure 1b). The cell viability data for LM1 ( $x p f)$ and LM4 ( $y G l A$ ) were consistent with the corresponding $\mathrm{OD}_{600}$ values and were in agreement as well with those of the wild-type

a

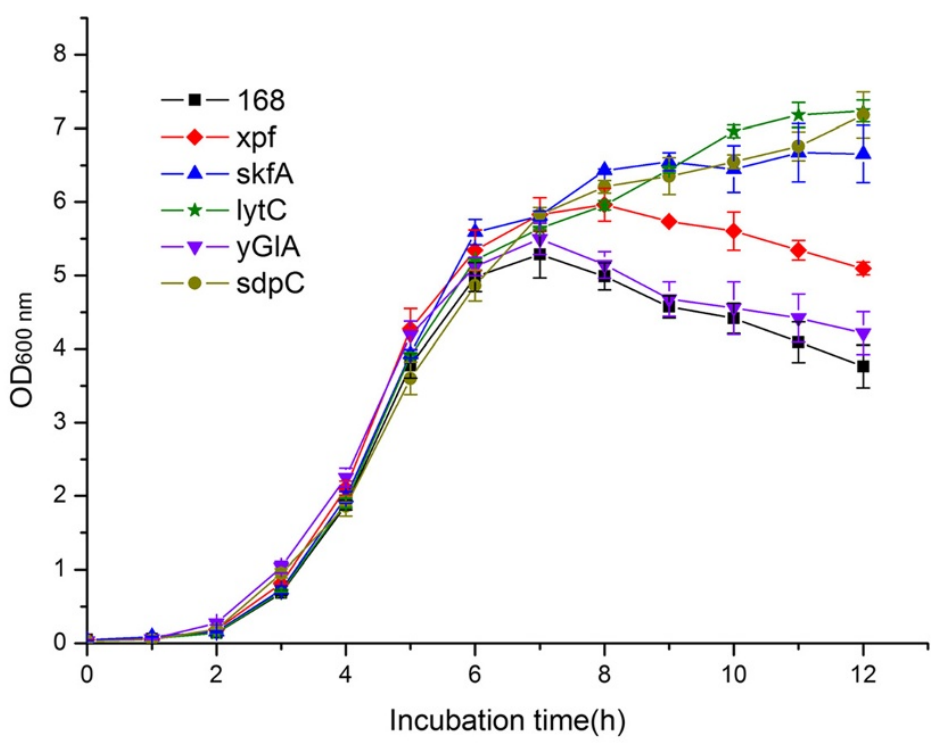

b

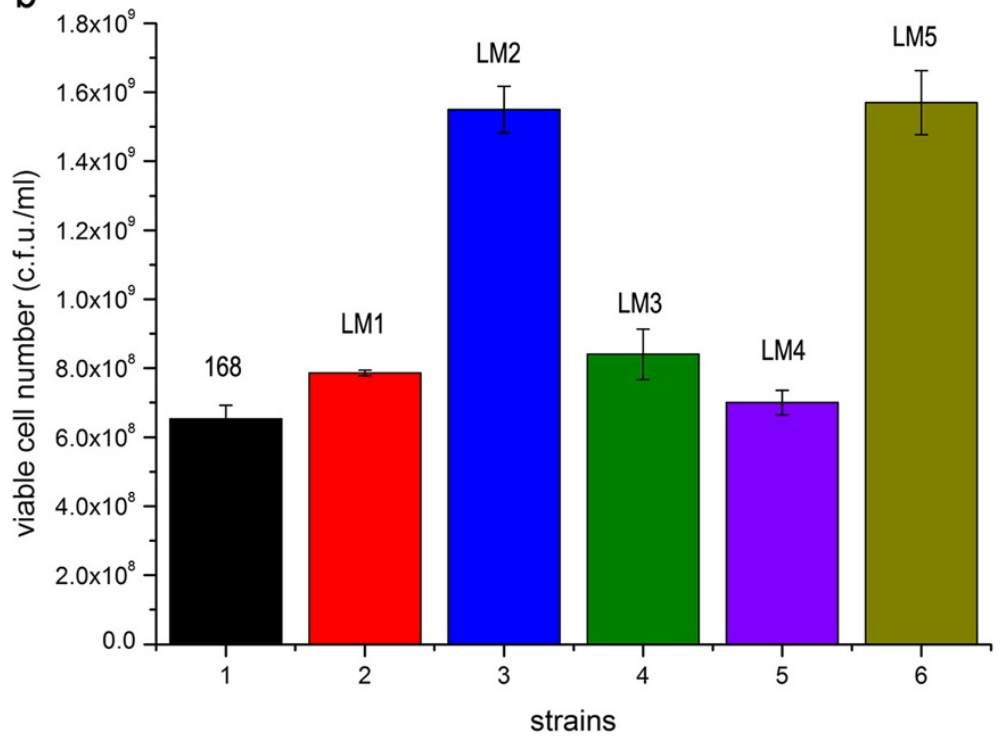

Figure 1 Biomass yields of single lytic-gene deletion mutants. (a) $\mathrm{OD}_{600}$ of cultures of wild-type and single-deletion mutants: $B$. subtilis 168 (Wild-type), $\bullet$ LM1, $\Delta L M 2, \star L M 3, \nabla L M 4, \bullet L M 5$. (b) 12-h viable cell numbers: 1 Wild-type, 2 LM1, 3 LM2, 4 LM3, 5 LM4, 6 LM5. The data presented represent the mean and standard deviation (SD) of triplicate measurements of three different colonies of the same genotype for each mutant. 
strain. In contrast, the high $\mathrm{OD}_{600}$ value of the LM3 $(l y t C)$ culture was not consistent with its viable count at $12 \mathrm{~h}$ (Figure 1a), which was only slightly higher than that of the parental strain (Figure 1b). To resolve this discrepancy, we observed cell morphology and found that mutants LM1 ( $x p f)$, LM2 (skfA), LM5 ( $s d p C)$, and LM4 (yGlA) appeared similar to the parental strain; however, LM3 (lytC) formed numerous chains (Additional file 1: Figure S2). Therefore, although the biomass of the $l y t C$ mutant increased compared with the parental strain, the formation of chains led to an underestimation of the number of viable cells. These results demonstrate that deleting $s k f A, l y t C, s d p C$, or $x p f$ significantly increased biomass compared with wild-type, although deletion of $y G l A$ had little effect.

\section{Analysis of biomass of $B$. subtilis strains harboring mutations in multiple genes encoding proteins involved in cell lysis}

We next generated six double-knockout strains as follows: LM12 ( xpf skfA), LM13 (xpf lytC), LM15 ( xpf sdpC), LM23 ( $s k f A$ lytC), LM25 (skfA sdpC), and LM35 (lytC $s d p C$ ). The biomass of each of the double mutants increased compared with that of the cognate single mutant after culture at $37^{\circ} \mathrm{C}$ for $12 \mathrm{~h}$ (Additional file 1 : Figure S3a). However, the influence of these double mutations was difficult to deduce from the performance of the respective single mutant. For example, we predicted that xpf would contribute the least to biomass compared with the other three strains; however, the $\mathrm{OD}_{600}$ of the LM15 $(x p f s d p C$ ) culture was the highest and exceeded that of LM23 (skfA lytC) by 14\%. Moreover, the numbers of viable cells of the double mutants were higher than that of their cognate single mutants, but not in complete agreement with the OD values. For example, although the $\mathrm{OD}_{600}$ of the LM25 ( $k f f A s d p C$ ) culture was not the highest, its viability was the highest and exceeded that of the parental strain by a factor of approximately 3 (Additional file 1: Figure S3b). Moreover, the viabilities of cultures of LM35 (lytC sdpC) and LM23 (skfA lytC) were lower when lytC was deleted compared with those of the other double mutants (Additional file 1: Figure S3b).

We next constructed two sets of mutants with multiple deletions in the order as follows: $s k f A, s d p C, l y t C$, $x p f, y G l A$ and $l y t C, s d p C, x p f$, skfA, $y G l A$. The growth curves and numbers of viable cells of the first series of mutants are shown in Figure 2. Although the $\mathrm{OD}_{600}$ value of the triple mutant (LM253) culture after $12 \mathrm{~h}$ was slightly higher than that of the double mutant (LM25), mutating more than two genes did not significantly increase biomass. The formation of chains by lytC deletion mutants once again caused an underestimation of the viable cell count based on the $\mathrm{OD}_{600}$ values (Additional file 1: Figure S4).

\section{Autolytic activity of deletion mutants}

Sodium azide is a cytochrome oxidase inhibitor that inhibits cell growth and it is used to assess the tendency of a cell to lyse under certain conditions [10]. When the $B$. subtilis strains were cultured with shaking in LB medium at $37^{\circ} \mathrm{C}$ for $3.5 \mathrm{~h}$ (mid-log phase), sodium azide was added to a final concentration of $0.05 \mathrm{M}$ and cell lysis was monitored by measuring $\mathrm{OD}_{600}$. Wild-type cells started to lyse immediately after sodium azide was added, and only $9 \%$ and $8 \%$ cells were intact after 3 and $4 \mathrm{~h}$, respectively. The results were similar for LM2 (skfA), LM5 $(s d p C)$, and LM4 $(y G l A)$. In contrast, $25 \%$ and $15 \%$ of the cells in the LM3 $(l y t C)$ culture were intact after 3 and $4 \mathrm{~h}$, respectively, in agreement with the results of previous studies $[10,31]$. Cell lysis was further reduced in cultures of LM1 (xpf) (35\% and 14\% of intact cells after 3 and $4 \mathrm{~h}$, respectively) (Figure 3a).

There were fewer lysed cells in cultures of the double mutants with deletions of $x p f$ or $l y t C$, namely LM12 (xpf skfA), LM13 ( $x p f l y t C$ ), LM15 ( $x p f s d p C$ ), LM23 (skfA lytC), and LM35 (lytC $s d p C$ ) in contrast to cultures of LM25 $(s k f A s d p C)$, which lysed to a similar extend compared with wild-type (Figure 3b).

Although the extents of cell lysis in cultures of the single mutant LM2 (skfA) and double mutant LM25 $(s k f A s d p C)$ were similar compared with wild-type, only $40 \%$ the cells in cultures of the triple mutant LM253 $(s k f A s d p C$ lytC) were lysed after $4 \mathrm{~h}$ (Figure 3c). Further, only $15 \%$ and $18 \%$ of the cells lysed after $4 \mathrm{~h}$ in cultures of the quadruple mutant LM2531 ( $s k f A s d p C$ lytC xpf) and quintuple mutant LM25314 ( $s k f A s d p C$ lytC xpf $y G l A)$. These observations indicate that deletion of $x p f$ and $l y t C$ exerted a significant effect on cell lysis.

The effects of the $s k f A$ and $s d p C$ mutations were significant as well. Although the extent of lysis in cultures of either of the single mutants LM2 (skfA) or LM5 $(s d p C)$ or the double mutant LM25 ( $s k f A s d p C)$ was not significantly altered, only $15 \%$ of the cells in cultures of the quadruple-mutant LM2531 ( $s k f A s d p C$ lytC xpf) were lysed after $4 \mathrm{~h}$ compared with $72 \%$ lysis in cultures of the double-mutant LM13 (xpf lytC) (Figure 3c). Moreover, cell lysis decreased when we deleted genes sequentially in the order $l y t C, s d p C, x p f$, skfA, and $y G l A$ as follows: wild-type, 92\%; single-mutant LM3 (lytC), 84\%; double-mutant LM35 (lytC $s d p C)$, 75\%; triple-mutant LM351 (lytC $s d p C$ xpf), 34\%; quadruple-mutant LM3512 (lytC sdpC xpf skfA), 15\%; and quintuple-mutant LM25314 (lytC sdpC xpf skfA yGlA), $10 \%$. These findings indicate that skfA or $s d p C$ exerted a combined influence on cell lysis when $x p f$ or $l y t C$ were mutated.

Therefore, we recommend a combined deletion of $l y t C$, $s d p C$, $x p f$, and $s k f A$ for constructing B. subtilis cell factories. Although the biomass of cultures of the quadruple mutant was similar to those of the double or triple mutants 
a
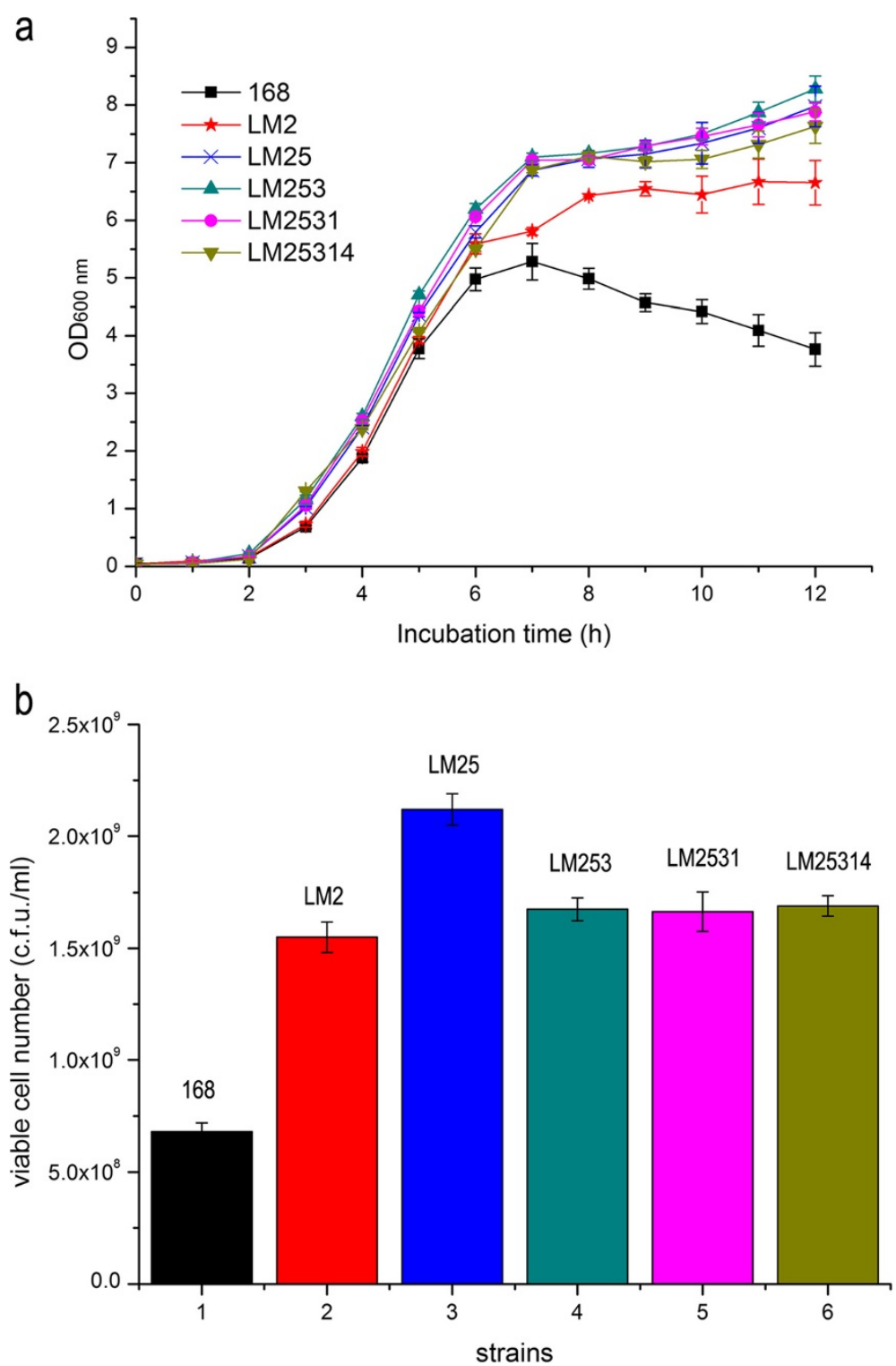

Figure 2 Biomass yields of multiple lytic-gene deletion mutants. (a) $\mathrm{OD}_{600}$ of cultures of wild-type and multiple-deletion mutants: Wild-type, $\star$ LM2, × LM25, $\boldsymbol{\Delta}$ LM253, • LM2531, $\boldsymbol{\nabla}$ LM25314; (b) 12 h viable cell numbers: 1 B. subtilis 168, 2 LM2, 3 LM25, 4 LM253, 5 LM2531, 6 LM25314. There was no significant difference between the biomass of each of the two sets of multiple-deletion mutants, and therefore data for one set is presented here. The data represent the mean and SD from triplicate measurements of three different colonies of the same genotype for each mutant.

(Figure 2), it exhibited increased resistance to lysis under adverse conditions (e.g. sodium azide) (Figure $3 \mathrm{c}$ and d). Deletion of $y G l A$ was the only mutation that did not detectably influence cell lysis (Figure 3) or biomass (Figures 1, 2).

\section{Production of recombinant proteins by the quadruple deletion mutant}

We next tested the ability of the quadruple mutant to express recombinant $\beta$-galactosidase and nattokinase by using the expression vectors $\mathrm{pBL}$ and $\mathrm{pBNA}$, respectively (Additional file 1: Appendix S1, Figure S1). There were similar levels of $\beta$-galactosidase in cell pellets of LM2531 and the wild-type during exponential growth $(<7 \mathrm{~h})$; however, they differed significantly upon entry into stationary phase, consistent with their biomass yields. The $\beta$-galactosidase activity of the wild-type peaked at $7 \mathrm{~h}$ and then declined, in contrast, to that of LM2531, which continued to increase after $7 \mathrm{~h}$, peaked at $13 \mathrm{~h}$ at a level 1.72 times higher than that of wild-type, and remained constant thereafter (at least until $18 \mathrm{~h}$ ). The results of SDS-PAGE analysis of $\beta$-galactosidase expression by cells harvested after $12 \mathrm{~h}$ of culture are consistent with the activity data (Figure 4). Note that, although the $\beta$-galactosidase encoded by pBL lacked a secretory signal sequence and 

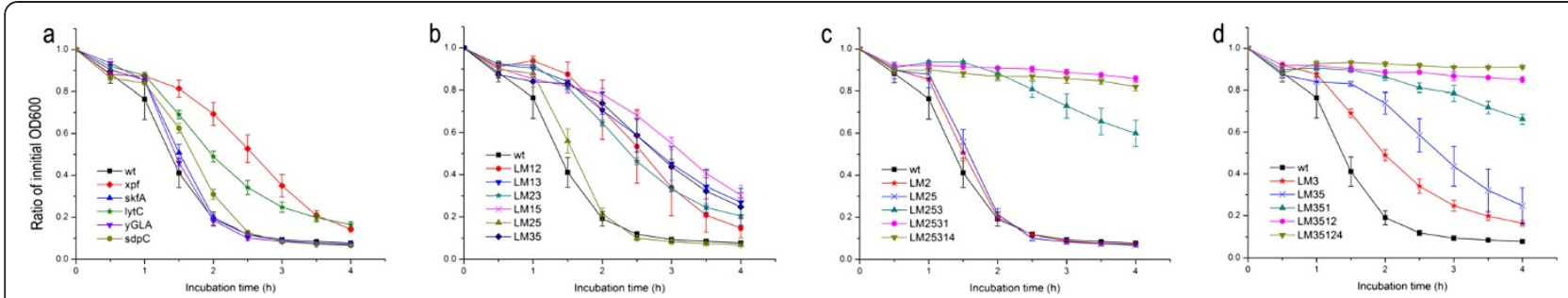

Figure 3 Effect of sodium azide on lysis of exponential-phase cultures. (a) Lysis of single mutants: $\mathbf{m i l d}$-type, $\bullet L M 1, \mathbf{\Delta} L M 2, \star L M 3, \boldsymbol{\nabla} L M 4$, - LM5. (b) Lysis of double mutants: wild-type, $\bullet$ LM12, $\boldsymbol{\nabla} L M 13, \star L M 23, \times L M 15, \boldsymbol{\Delta} L M 25,-L M 35$. (c) First set of multiple mutants: wild-type, $\star$ LM2, × LM25, $\boldsymbol{\Delta}$ LM253, • LM2531, $\boldsymbol{\nabla}$ LM25314. (d) Second set of multiple mutants: wild-type, $\star L M 3, \times L M 35, \boldsymbol{\Delta} L M 351, \bullet L M 3512, \boldsymbol{\nabla}$ LM35124. The data represent the mean and SD from triplicate measurements of three different colonies of the same genotype for each mutant.

therefore expressed intracellularly (Additional file 1: Appendix S1, Figure S1), $\beta$-galactosidase activity was detected in the supernatants of cultures of both strains, particularly in stationary phase, likely due to cell lysis. However, $\beta$-galactosidase activity in the supernatant of $168 / \mathrm{pBL}$ was significantly higher than that of LM2531/ pBL at $12 \mathrm{~h}$, indicating significant inhibition of cell lysis of the quadruple-deletion mutant.

Because the ability to secrete recombinant proteins is a major advantage of using $B$. subtilis, we transformed the quadruple-mutant LM2531 with a plasmid (pBNA) expressing nattokinase containing a secretory signal peptide. The supernatants and cells harvested from cultures of LM2531 and wild-type, each transformed with pBNA, were assayed for fibrinolysis catalyzed by nattokinase (Figure 5a). Fibrinolytic activity was detected only in supernatants of both cultures and was higher by a factor of 2.6 in supernatants of LM2531 cultures (Figure 5b). The expression of nattokinase in culture supernatants harvested at 6 (log phase), 12 (stationary phase), and $24 \mathrm{~h}$ (death phase) was analyzed using SDS-PAGE (Figure 5c). A major band of approximately $29 \mathrm{kDa}$ corresponding to mature nattokinase [36] was detected at each sampling time (lanes 3-8) but not in cultures of untransformed cells (lanes 1 and 2). The amounts of nattokinase in LM2531 supernatants (lanes 4, 6, 8) were greater compared with those of the wild-type (lanes 3, 5, 7). Moreover, the total amount of wild-type proteins in the supernatant increased from 6 to 24 h (lanes 4, 6, 8), indicating increased cell lysis compared with mutant cultures.

\section{Discussion}

Lysis of B. subtilis strains used for industrial purposes significantly decreases product yield $[7,37]$. We addressed

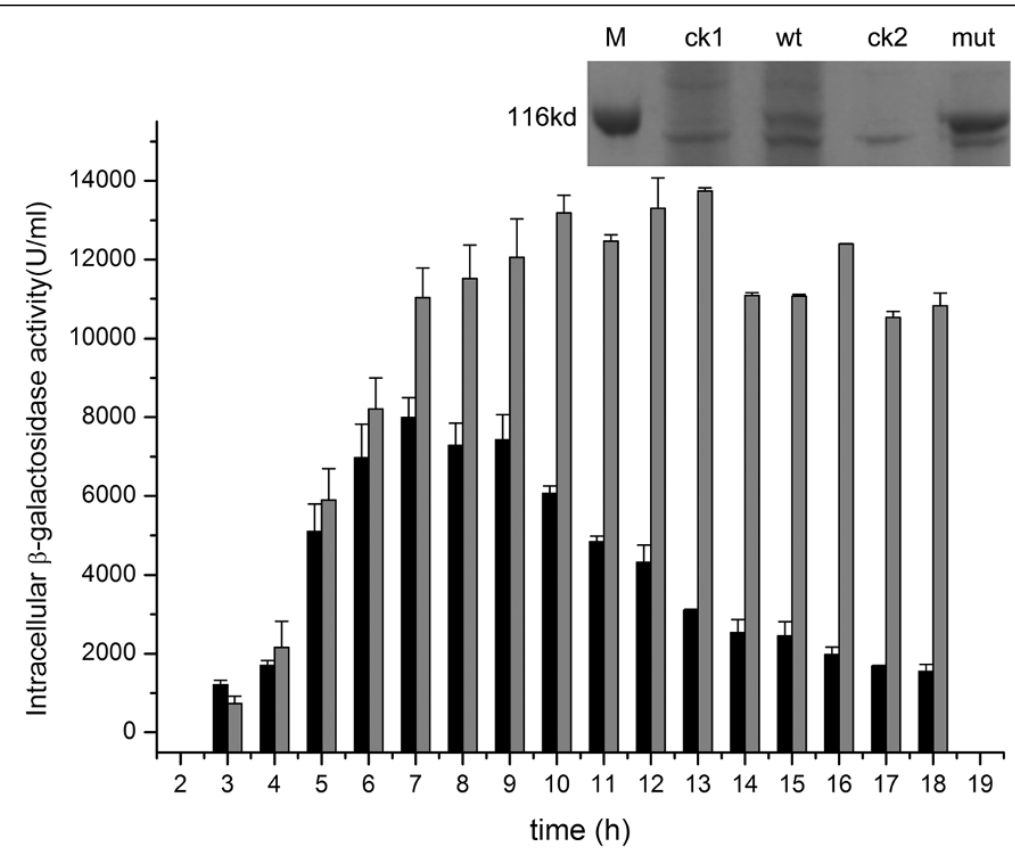

Figure 4 Intracellular production of $\beta$-galactosidase. Wild-type (wt) (black columns), LM2531 (mut) (gray columns). Inset: SDS-PAGE analysis of cell pellets. Standard (M), B. subtilis 168/pBE2 (ck1), B. subtilis 168/pBL (wt), LM2531/pBE2 (ck2), LM2531/pBL (mut). 

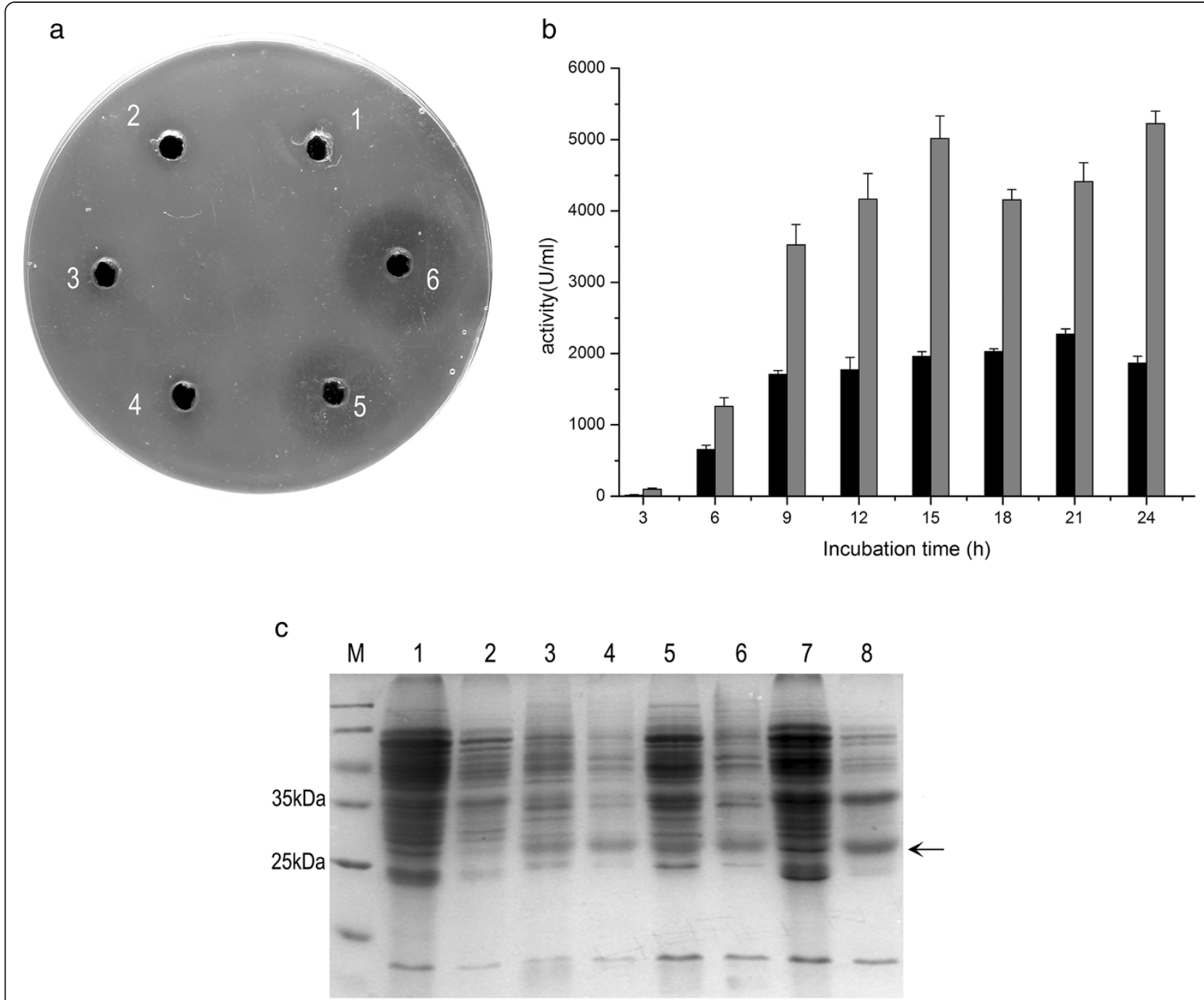

Figure 5 Nattokinase production in supernatants of wild-type and mutant cultures. (a) Nattokinase activity in the supernatants of wild-type and mutant cultures, Wild-type (wt) (black columns), LM2531 (mut) (gray columns). (b) SDS-PAGE analysis. 1. B. subtilis 168/pBE2 supernatant, 2. LM2531/pBE2 supernatant as control, 3. B. subtilis 168/pBNA supernatant at 6 h, 4. LM2531/pBNA supernatant at 6 h, 5. B. subtilis 168/pBNA supernatant at $12 \mathrm{~h}, 6$. LM2531/pBNA supernatant at $24 \mathrm{~h}, 7$. B. subtilis 168/pBNA supernatant at $24 \mathrm{~h}, 8$. LM2531/pBNA supernatant at $24 \mathrm{~h}$. The arrow indicates the position corresponding to mature nattokinase $(29 \mathrm{kDa})$. (c): fibrinolytic activity assay. 1. B. subtilis 168/pBE2 supernatant, 2. LM2531/pBE2 supernatant, 3. B. subtilis 168/pBNA cell lysate, 4. LM2531/pBNA cell lysate, 5. B. subtilis 168/pBNA supernatant, 6. LM2531/pBNA supernatant.

this issue here by systematically deleting genes involved in autolysis from the wild-type strain B. subtilis 168 and assessed the effects of single and multiple deletions on cell lysis and biomass production.

SkfA and SdpC are sibling-killing factors that are implicated in the sporulation of B. subtilis $[15,16]$; however, their role in other growth phases is unknown. We show here that $s k f A$ and $s d p C$ were transcribed during $\log$ phase (Additional file 1: Figure S5), which is consistent with our findings that cell lysis was simultaneously attenuated (Figure 3). However, the effect of deleting $s k f A$ and $s d p C$ was only detected when $x p f$ or $l y t C$ were also deleted, suggesting that their expression levels were relatively low and therefore undetectable when $x p f$ and
lytC are expressed by the wild-type. We reasoned that the effects of lytic enzymes might be redundant. Therefore, we constructed mutants with multiple deletions of lytic genes. The highest numbers of intact cells were detected in cultures of the quadruple-mutant LM3512 $(l y t C s d p C$ $x p f s k f A$ ). However, deleting $s k f A$ or $s d p C$ from LM13 (xpf $l y t C$ ) did not cause a further increase in biomass (Figure 2), suggesting that the balance between cell lysis and cell growth is very complex and requires further investigation.

Lysis of B. subtilis is mediated by the products of prophage genes. Although the defective prophage PBSX does not influence the growth, proliferation, or sporulation of B. subtilis $[38,39]$ we show here that the sigma-like prophage PBSX factor Xpf is involved in the normal growth 
and differentiation of B. subtilis. Although $x p f$ was deleted from the LM1 mutant, cell lysis was effectively decreased, it produced more spores than wild-type (Additional file 1: Figure S6A). To the best of our knowledge, this is the first evidence that links this defective prophage to cell lysis and biomass accumulation in Bacillus. The prophage skin element harbors phage-like operon sequences that are highly homologous to segments of the regulatory region and late operon of PBSX, but there are 17 more ORFs with the region of skin since significant rearrangements. Unlike PBSX, skin cannot be induced by SOS response [40]. Our present results confirm speculation that this prophage is not active in B. subtilis [40], because all the mutations of $y G l A$ exhibited no obvious effects on biomass yield or cell autolysis.

PG hydrolases represent a distinct category of lytic enzymes. LytC is the major vegetative PG hydrolase expressed by $B$. subtilis, and it mediates cell separation, cell wall turnover, antibiotic-induced lysis, and motility [24]. Here we show that the deletion mutant of $l y t C$ (LM3) was more resistant to cell lysis compared with wild-type and formed chains, in contrast to the wild-type and other mutant strains (Additional file 1: Figure S2).

Because inactivating PG hydrolases is not lethal, it was possible to construct deletion mutants. PG hydrolases such as LytD, LytE, and LytG disrupt the cell wall in vitro $[24,25,27]$. However, we did not delete them all, because lack of PG hydrolase activity may interfere with the ability of cells to separate or decrease membrane permeability, which decreases protein yields [11]. Therefore, a balance between resistance to lysis and efficient protein expression is required. The mutant LM2531 performed well in this regard.

Although there are published studies on the relationship between certain lytic genes and biomass production, none addresses the combined effects of the products of these genes on cell lysis and biomass yield. We demonstrate here that cultures of the quadruple mutant LM2531 exhibited the least cell lysis and high biomass yield compared with wild-type. Moreover, LM2531 expressed significant higher levels of intracellular recombinant $\beta$-galactosidase for a prolonged period and secreted recombinant nattokinase compared with the wild type strain.

We believe that the enhanced resistance of LM2531 to cell lysis and its ability to efficiently express recombinant proteins may be attributed to its production of increased numbers of cells. Although the amount of protein correlates with cell number, there are exceptions. For example, individual silencing of $l y t C$ and SpoIIGA in the Bacillus strain ATCC 6051 increased cell densities; however, only the lytC mutant produced increased levels of alphaamylase [41]. SpolIGA is a membrane-associated aspartic protease responsible for activating the early sporulation sigma factor E [42]. Therefore, we reasoned that the production of recombinant protein might be optimized before spoIIGA is transcribed. Thus, vegetative genes may be a better choice for this purpose. This assumption is supported by the outcome of deleting four genes from the wild-type that are expressed during the vegetative phase.

A second explanation to account for the phenotype of LM2531 is the deletion of genes that prevent sporulation. Thus, after $12 \mathrm{~h}$, approximately $50 \%$ of the wild-type cells formed spores, in contrast to less than $10 \%$ in cultures of the quadruple mutant (Additional file 1: Figure S6B). The presence of spores reduces the yield of target products and increase production costs, because it is difficult to remove spores from bioreactors [43]. Therefore, inhibiting spore formation will likely benefit the production of recombinant proteins.

Third, the inactivation of lysis genes inhibited leakage of cellular contents during cell lysis. For example, the inactivation of lytC prevents the leakage of intracellular proteins into the culture fluid [31]. Consistent with these results, we show here that the amount of total extracellular proteins was high in wild-type cultures. In contrast, they were present at extremely low levels in LM2531 cultures (Figure 5c). Cell lysis releases proteases into the culture fluid that may degrade secreted recombinant proteins. Therefore, inhibiting cell lysis may lead to higher yields of recombinant protein produced by the mutant. Moreover, minimizing the amount of degradation products and materials released by lysed cells will simplify downstream purification processes.

\section{Conclusions}

Here we implemented a new strategy to enhance biomass yield and prevent cell autolysis in liquid cultures of wildtype B. subtilis 168 by creating mutants with deletions of multiple lytic genes. The production of intracellular and secreted recombinant proteins was significantly elevated in cultures of the mutant LM2531 by the deletion of $l y t \mathrm{C}$, which encodes a major PG hydrolase; prophage-encoded $x p f$; and cannibalism factors $s k f A$ and $s d p C$. Because these lytic genes are conserved among Bacillus species, the results of our experiments performed here using $B$. subtilis 168 will provide a guideline for genetic manipulations of other Bacillus strains to enhance the production of industrial materials.

\section{Methods}

\section{Strains and plasmids}

All strains and plasmids used in this study are listed in Additional file 1: Tables S1 and Additional file 1: Table S2. $B$. subtilis mutants were derived from wild-type $B$. subtilis 168 (CCTCC AB93017). Escherichia coli DH5 $\alpha$ was used as the host for plasmid construction. The E. coli/ B. subtilis shuttle vector pNNB194 [44] was used to generate gene deletions and replacements. Plasmid pBE2 was 
used to construct vectors for expressing recombinant proteins [45]. Genetic procedures for constructing plasmids and mutants are shown in Additional file 1: Appendix S1.

\section{Measurement of biomass and cell viability}

The biomass of $B$. subtilis cultures was evaluated according to optical density (OD) and viable cell number. Overnight cultures of wild-type and mutants were inoculated into LB medium at a dilution of $1: 100$ and shaken on a reciprocating shaker at $200 \mathrm{rpm}$ at $37^{\circ} \mathrm{C}$ for $12 \mathrm{~h}$. The OD at $600 \mathrm{~nm}$ was measured at $1 \mathrm{~h}$ intervals, and serial dilutions of $12-\mathrm{h}$ cultures were plated onto LB agar. The numbers of colonies were counted after incubation for $16 \mathrm{~h}$. All the experiments were performed in triplicate using three different colonies of the same genotype.

\section{Analysis of spore formation}

The number of spores was evaluated by colony counts after heat-shock treatment of $80^{\circ} \mathrm{C}$ for $20 \mathrm{~min}$ followed by immediate cooling in water to room temperature. Serially diluted samples $(100 \mu \mathrm{l})$ were spread on LB plates. After the plates were incubated at $37^{\circ} \mathrm{C}$ for $16 \mathrm{~h}$, the number of colonies was used to calculate the number of spores in the sample. All the experiments were performed in triplicate using three different colonies of the same genotype.

\section{Measurement of cell autolysis}

Wild-type and mutants were grown to mid-exponential phase $(3.5 \mathrm{~h})$ in LB medium. After adding sodium azide to a final concentration $0.05 \mathrm{M}(\mathrm{MIC} \times 10)$, the $\mathrm{OD}_{600}$ of the cultures was measured at $30-\mathrm{min}$ intervals at $37^{\circ} \mathrm{C}$ and $200 \mathrm{rpm}$. The ratio of $\mathrm{OD}_{600}$ at each sampling time to the initial $\mathrm{OD}_{600}$ was used to calculate cell lysis $[10,46]$. All the experiments were performed in triplicate using three different colonies of the same genotype.

\section{$\beta$-Galactosidase assays}

Recombinant $B$. subtilis strains carrying $\mathrm{pBL}$ were grown in LB medium supplemented with $10 \mu \mathrm{g} / \mathrm{ml}$ of kanamycin. Samples $(0.2 \mathrm{ml})$ were taken at $1 \mathrm{~h}$ intervals and centrifuged. The pellets were resuspended in $1 \mathrm{ml}$ of $\mathrm{Z}$ buffer [47] containing $0.1 \mathrm{mg} / \mathrm{ml}$ of lysozyme, and incubated at $37^{\circ} \mathrm{C}$ for $20 \mathrm{~min}$ to remove cell walls. The cells were permeabilized by adding $0.1 \%$ Triton $\mathrm{X}-100$, one drop of $0.1 \%$ SDS, and two drops of chloroform. After equilibrating the tubes in a $30^{\circ} \mathrm{C}$ water bath for $5 \mathrm{~min}, 0.2 \mathrm{ml}$ ONPG was added to each tube. After incubation at $30^{\circ} \mathrm{C}$ for $2 \mathrm{~min}$, the reaction was stopped by adding $0.5 \mathrm{ml}$ of $1 \mathrm{M} \mathrm{Na}_{2} \mathrm{CO}_{3}$. Absorbance was recorded at $420 \mathrm{~nm}$ using a spectrophotometer. $\beta$-Galactosidase activity was defined as the amount of enzyme required to release $1 \mathrm{nmol}$ of 2-nitrophenol from ONPG per min in $1 \mathrm{ml}$ of culture fluid. The equation to calculate $\beta$-galactosidase activity $[47,48]$ is as follows:

$$
\text { Activity }=\frac{\mathrm{OD} 420}{\mathrm{t} \times \mathrm{vol}} \times \frac{1 \mathrm{nmol}}{0.0045 \mathrm{ml} \mathrm{cm}} \times 1.7 \mathrm{ml}
$$

Where $\mathrm{t} \min =$ reaction time $(2 \mathrm{~min})$

vol $\mathrm{ml}=$ volume of culture

$0.0045 \mathrm{OD}_{420} / \mathrm{nmol}=$ extinction coefficient of 2nitrophenol

$1.7 \mathrm{ml}=$ total volume of reaction mixture

cuvette $=1 \mathrm{~cm}$ path length

Activity $=\mathrm{nmol} / \mathrm{min} / \mathrm{ml}=\mathrm{U} / \mathrm{ml}$

Cell pellets (approximately $100 \mu \mathrm{l}$ ) were disrupted using an ultrasonicator and were analyzed using SDS-PAGE.

\section{Fibrinolytic enzyme assays}

Nattokinase was first identified in the Japanese fermented food natto. It is widely used to preventing thrombotic diseases because it is a food and exerts strong fibrinolytic activity [49]. We PCR-amplified the gene encoding nattokinase from $B$. natto strain BN13. The amplicon contained an open reading frame (ORF) of 1143 bp starting with GTG that was predicted to encode a 29-residue secretory signal peptide, a 77-residue propeptide, and a 275-residue mature enzyme (approximately $29 \mathrm{kDa}$ ) [36]. The entire ORF was used to construct plasmid pBNA (For details, Additional file 1: Appendix S1).

B. subtilis strains transformed with pBNA were grown in LB medium supplemented with $10 \mu \mathrm{g} / \mathrm{ml}$ of kanamycin. Samples were taken at 3-h intervals. After centrifugation, cell pellets and supernatants were collected. The pellets were washed twice with phosphate-buffered saline (PBS), resuspended into the same volume of PBS buffer, and disrupted using an ultrasonicator. Fibrinolytic activity was determined by measuring the areas of the clear zone on the fibrin plates, which were prepared according to Astrup and Sterndorff [50]. The supernatant or disrupted cell suspension (10 $\mu \mathrm{l}$ each) was added to each hole. Using commercial urokinase as a standard, we generated a standard curve (see Additional file 1: Figure S7) to determine fibrinolytic activity. Approximately $500 \mu \mathrm{l}$ of supernatant or $100 \mu \mathrm{l}$ of sonicated cell pellets was analyzed using SDS-PAGE.

\section{Additional file}

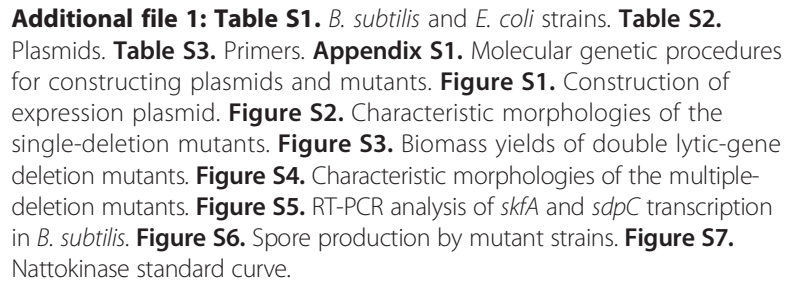

Abbreviations

yGIA: Gene cluster yqxG-yqxH-cWIA; PG: Peptidoglycan. 


\section{Competing interests}

The authors declare that they have no competing interests.

\section{Authors' contributions}

Conception and design of the study: XD Chen. Acquisition of data: Y Wang, $\Pi \mathrm{T}$ Jin, RL Zhao and XM Zhang. Analysis and interpretation of data: Y Wang and ZM Chen. Drafting the article: Y Wang. Revising it critically for important intellectual content: XD Chen and ZM Chen. Final approval of the version to be submitted: all co-authors. All authors read and approved the final manuscript.

\section{Acknowledgments}

We thank Dr. Mariah Bindel Connelly (Novozymes, Inc., 1445 Drew Ave., Davis, CA 95618, United States), who kindly provided us the plasmid pNNB194. This work was supported by grants from the National Basic Research Program of China (973 Program) (No. 2011CB808800) and the National Natural Science Foundation of China (No. 31270145).

\section{Author details}

${ }^{1}$ State Key Laboratory of Virology, College of Life Sciences, Wuhan University, Wuhan 430072, China. ${ }^{2}$ State Key Laboratory of Agricultural Microbiology, Huazhong Agricultural University, Wuhan, China. ${ }^{3}$ Hubei Provincial Cooperative Innovation Center of Industrial Fermentation, Wuhan 430072, China.

Received: 13 May 2014 Accepted: 23 August 2014 Published: 31 August 2014

\section{References}

1. Harwood CR: Bacillus subtilis and its relatives: molecular biological and industrial workhorses. Trends Biotechnol 1992, 10:247-256.

2. Hong HA, Duc LH, Cutting SM: The use of bacterial spore formers as probiotics. FEMS Microbiol Rev 2005, 29(4):813-835.

3. Senesi S: Bacillus spores as probiotics products for human use. In Bacterial spores: Probiotics and Emerging Applications. London: Horizon Science Press; 2004:132-141.

4. Emmert EAB, Handelsman J: Biocontrol of plant disease-a (Gram-) positive perspective. FEMS Microbiol Lett 1999, 171:1-9.

5. Montesinos E: Development, registration and commercialization of microbial pesticides for plant protection. Int Microbiol 2003, 6:245-252.

6. Shoda M: Bacterial control of plant diseases. J Biosci Bioeng 2000, 89:515-521.

7. Schallmey M, Singh A, Ward OP: Developments in the use of Bacillus species for industrial production. Can J Microbiol 2004, 50(1):1-17.

8. van Dijl H: Bacillus subtilis: from soil bacterium to super-secreting cell factory. Microb Cell Fact 2013, 12:3.

9. Zhang $H$, Wang $Y$, Pfeifer BA: Bacterial hosts for natural product production. Mol Pharm 2008, 5(2):212-225.

10. Smith TJ, Blackman SA, Foster SJ: Autolysins of Bacillus subtilis: multiple enzymes with multiple functions. Microbiology 2000, 146(2):249-262.

11. Van Heijenoort J: Peptidoglycan hydrolases of Escherichia coli. Microbiol Mol Biol Rev 2011, 75(4):636-663.

12. Vollmer W, Joris B, Charlier P, Foster S: Bacterial peptidoglycan (murein) hydrolases. FEMS Microbiol Rev 2008, 32(2):259-286.

13. Engelberg-Kulka H, Hazan R: Cannibals defy starvation and avoid sporulation. Sci (Washington) 2003, 301(5632):467-468.

14. Engelberg-Kulka H, Amitai S, Kolodkin-Gal I, Hazan R: Bacterial programmed cell death and multicellular behavior in bacteria. PLoS Genet 2006, 2(10):e135.

15. González-Pastor JE, Hobbs EC, Losick R: Cannibalism by sporulating bacteria. Science 2003, 301(5632):510-513.

16. Westers H, Braun PG, Westers L, Antelmann H, Hecker M, Jongbloed JD, Quax WJ: Genes involved in SkfA killing factor production protect a Bacillus subtilis lipase against proteolysis. Appl Environ Microbiol 2005, 71(4):1899-1908.

17. Kunst F, Ogasawara N, Moszer I, Albertini AM, Alloni GO, Azevedo V, Haga K: The complete genome sequence of the gram-positive bacterium Bacillus subtilis. Nature 1997, 390(6657):249-256

18. Regamey A, Karamata D: The N-acetylmuramoyl-I-alanine amidase encoded by the Bacillus subtilis 168 prophage SP $\beta$. Microbiology 1998, 144(4):885-893.
19. Rosenthal R, Toye PA, Korman RZ, Zahler SA: The prophage of SP $\beta$ C2dcitK1, a defective specialized transducing phage of Bacillus subtilis. Genetics 1979, 92(3):721-739.

20. Lazarevic V, Düsterhöft A, Soldo B, Hilbert H, Mauël C, Karamata D: Nucleotide sequence of the Bacillus subtilis temperate bacteriophage SPßC2. Microbiology 1999, 145(5):1055-1067.

21. Wood HE, Dawson MT, Devine KM, McCONNELL DJ: Characterization of PBSX, a defective prophage of Bacillus subtilis. J Bacteriol 1990, 172(5):2667-2674.

22. Shingaki R, Kasahara $Y$, Inoue T, Kokeguchi S, Fukui K: Chromosome DNA fragmentation and excretion caused by defective prophage gene expression in the early-exponential-phase culture of Bacillus subtilis. Can J Microbiol 2003, 49(5):313-325.

23. Foster SJ: Cloning, expression, sequence analysis and biochemical characterization of an autolytic amidase of Bacillus subtilis $168 \operatorname{trpC2}$. J Gen Microbiol 1991, 137(8):1987-1998.

24. Blackman SA, Smith TJ, Foster SJ: The role of autolysins during vegetative growth of Bacillus subtilis 168. Microbiology 1998, 144(1):73-82.

25. Margot $P$, Wahlen $M$, Gholamhuseinian A, Piggot $P$, Karamata D: The lytE gene of Bacillus subtilis 168 encodes a cell wall hydrolase. J Bacterio 1998, 180(3):749-752.

26. Ohnishi R, Ishikawa S, Sekiguchi J: Peptidoglycan hydrolase LytF plays a role in cell separation with CwIF during vegetative growth of Bacillus subtilis. J Bacterio/ 1999, 181(10):3178-3184.

27. Horsburgh GJ, Atrih A, Williamson MP, Foster SJ: LytG of Bacillus subtilis is a novel peptidoglycan hydrolase: the major active glucosaminidase. Biochemistry 2003, 42(2):257-264.

28. Yamaguchi $H$, Furuhata $K$, Fukushima T, Yamamoto $H$, Sekiguchi J: Characterization of a new Bacillus subtilis peptidoglycan hydrolase gene $y v c E$ (named $c w / O$ ), and the enzymatic properties of its encoded protein. J Biosci Bioeng 2004, 98(3):174-181.

29. Fukushima T, Afkham A, Kurosawa SI, Tanabe T, Yamamoto H, Sekiguchi J: A new D, L-endopeptidase gene product, YojL (renamed CWIS), plays a role in cell separation with LytE and LytF in Bacillus subtilis. J Bacteriol 2006, 188(15):5541-5550

30. Fukushima T, Yao Y, Kitajima T, Yamamoto H, Sekiguchi J: Characterization of new L, D-endopeptidase gene product CwIK (previous YcdD) that hydrolyzes peptidoglycan in Bacillus subtilis. Mol Genet Genomics 2007, 278:371-383.

31. Kodama T, Endo K, Ara K, Ozaki K, Kakeshita H, Yamane K, Sekiguchi J: Effect of Bacillus subtilis spoOA mutation on cell wall lytic enzymes and extracellular proteases, and prevention of cell lysis. J Biosci Bioeng 2007, 103(1):13-21.

32. Bisicchia P, Noone D, Lioliou E, Howell A, Quigley S, Jarmer TJH, Devine KM: The essential YycFG two-component system controls cell wall metabolism in Bacillus subtilis. Mol Microbiol 2007, 65:180-200.

33. Hashimoto M, Ooiwa S, Sekiguchi J: Synthetic lethality of the lytE cwlO genotype in bacillus subtilis is caused by lack of $D$, L-endopeptidase activity at the lateral cell wall. J Bacterio/ 2012, 194(4):796.

34. McDonnell GE, McConnell DJ: Overproduction, isolation, and DNA-binding characteristics of Xre, the repressor protein from the Bacillus subtilis defective prophage PBSX. J Bacteriol 1994, 176(18):5831-5834.

35. Stephenson K, Bron S, Harwood CR: Cellular lysis in Bacillus subtilis; the affect of multiple extracellular protease deficiencies. Lett App/ Microbiol 1999, 29(2):141-145.

36. Nakamura T, Yamagata Y, Ichishima E: Nucleotide sequence of the subtilisin NAT gene, aprN, of Bacillus subtilis (natto). Biosci Biotechnol Biochem 1992, 56(11):1869-1871.

37. Iyer JL, Shetty P, Pai JS: Evaluation of whole cells of Bacillus subtilis as substrate for measurement of autolysin activity. Process Biochem 2005, 40:1593-1597

38. Westers H, Dorenbos R, van Dijl JM, Kabel J, Flanagan T, Devine KM, Quax WJ: Genome engineering reveals large dispensable regions in Bacillus subtilis. Mol Biol Evol 2003, 20(12):2076-2090.

39. Ara K, Ozaki K, Nakamura K, Yamane K, Sekiguchi J, Ogasawara N: Bacillus minimum genome factory: effective utilization of microbial genome information. Biotechnol Appl Biochem 2007, 46(3):169-178.

40. Krogh S, O'Reilly M, Nolan N, Devine KM: The phage-like element PBSX and part of the skin element, which are resident at different locations on the Bacillus subtilis chromosome, are highly homologous. Microbiology 1996, 142(8):2031-2040. 
41. Kabisch J, Thürmer A, Hübel T, Popper L, Daniel R, Schweder T: Characterization and optimization of Bacillus subtilis ATCC 6051 as an expression host. J Biotechnol 2013, 163(2):97-104.

42. Imamura D, Kuwana R, Kroos L, Feig M, Takamatsu H, Watabe K: Substrate specificity of SpollGA, a signal-transducing aspartic protease in Bacilli. J Biochem 2011, 149:665-671.

43. Spilimbergo S, Bertucco A, Lauro FM, Bertoloni G: Inactivation of Bacillus subtilis spores by supercritical $\mathrm{CO} 2$ treatment. Innovat Food Sci Emerg Tech 2003, 4:161-165.

44. Connelly MB, Young GM, Sloma A: Extracellular proteolytic activity plays a central role in swarming motility in Bacillus subtilis. J Bacteriol 2004, 186 (13):4159-4167.

45. Guo X, Xiong Z, Jia $S, X u Y$ : The construction of multifunctional shuttle vectors of Bacillus subtilis- Escherichia coli. Chin J Biotech 1991, 7:224-229.

46. Tomasz A, Albino A, Zanati E: Multiple antibiotic resistance in a bacterium with suppressed autolytic system. Nature 1970, 227:138-140.

47. Miller JH: Experiments in molecular genetics. In Cold Spring Harbor N.Y: Cold Spring Harbdr Laboratory; 1972.

48. Ferrari E, Howard SM, Hoch JA: Effect of stage 0 sporulation mutations on subtilisin expression. J Bacteriol 1986, 166(1):173-179.

49. Sumi $\mathrm{H}$, Hamada $\mathrm{H}$, Tsushima $\mathrm{H}$, Mihara H, Muraki $\mathrm{H}$ : A novel fibrinolytic enzyme (nattokinase) in the vegetable cheese Natto; a typical and popular soybean food in the Japanese diet. Experientia 1987, 43(10):1110-1111.

50. Astrup T, Müllertz S: The fibrin plate method for estimating fibrinolytic activity. Arch Biochem Biophys 1952, 40(2):346-351.

doi:10.1186/s12934-014-0129-9

Cite this article as: Wang et al:: Deleting multiple lytic genes enhances biomass yield and production of recombinant proteins by Bacillus subtilis. Microbial Cell Factories 2014 13:129.

\section{Submit your next manuscript to BioMed Central and take full advantage of:}

- Convenient online submission

- Thorough peer review

- No space constraints or color figure charges

- Immediate publication on acceptance

- Inclusion in PubMed, CAS, Scopus and Google Scholar

- Research which is freely available for redistribution 